\title{
Sensoric and motor recovery of cranial nerves: a case report
}

\author{
R. Slavicek ${ }^{1}$, U. Reckewert ${ }^{2}$, G. Slavicek ${ }^{3}$ \\ ${ }^{1}$ Department for Interdisciplinary Dentistry and Technology, Danube University Krems, Krems, Austria \\ ${ }^{2}$ Privat Practice, Potsdam, Germany \\ ${ }^{3}$ Steinbeis Transfer Institut Biotechnology in Interdisciplinary Dentistry der Steinbeis Hochschule Berlin, Stuttgart, Germany
}

Received November 20, 2008; Accepted January 10, 2009

\begin{abstract}
A case of neurological complication after surgical procedure for a Meningeoma of the left cavernous sinus is presented. It concerns a healthy 27-year-old woman who developed left side facial muscle atrophy in combination with dysesthesia and hypalgesia in the left face. The diagnostic and therapeutical approach is described in detail. Adequate and functionally orientated diagnostic procedures are mandatory to establish an individual treatment plan. An interdisciplinary treatment, clearly focused on the recovery of the functions of the stomatognathic system, restores functions and improves local muscular and neuronal properties. In addition, the stricken psychic situation of the affected patient can be considerably improved by this approach. A route of capitulation should not be applied in such situations. An active and previsional treatment concept has to be implemented, although the actions taken have to be arranged on an individual base.
\end{abstract}

Keywords: Meningioma, neuronal complication, interdisciplinary treatment, EMG, muscle atrophy, jaw joint, trigeminal nerve palsy

\section{Introduction}

Meningiomas are usually benign tumors, located either intracranial or within the spinal canal. The source tissue is the arachnoid mater, one of the three meninges. This explains the common location on the brain surface, but in rare cases, intraventricular location can be observed. Still, the classification of meningiomas is controversially discussed, mainly because of the possibility of mesenchymal and epithelial occurrence. The WHO classification classifies grad I to III [5].

Incidence rates of symptomatic meningiomas are approximately 2 per 100,000 individuals and contribute approximately $25 \%$ of all primary intracranial neoplasm $[1,2]$. Females are affected more often (male to female ratio 1:2.8); the incidence rate increases with age [5].

Correspondence: Rudolf Slavicek, Department for Interdisciplinary Dentistry and Technology, Danube University Krems, Krems, Austria. E-mail: rudolf.slavicek@donau-uni.ac.at
The number of undiagnosed asymptomatic meningiomas is quite high. Up to $40 \%$ of the diagnosed meningeomas are multiple [6].

Slow growth is a main characteristic of meningeomas; the morbidity and symptoms might become dramatic, before causing death. The 5-year survival rates range from 70 to over $90 \%$.

A high postoperative morbidity can be predicted in connection with co-morbidities, elderly patients and poor pre-operative neurological status. Tumor criteria such as location, size, consistency and perfusion have to be taken into consideration as well, when estimating the prognosis of the individual patient.

Symptoms are different depending on the size and location. Irritation and compression of brain and cranial nerves and interruption of vascular flow are commonly accepted models of mechanisms. The wide range of neurological symptoms [7] makes clinical diagnosis difficult. Headache, vomiting, gait disturbance, ocular symptoms, fatigue and seizures are most common. But tics, weakness, numbness, tremor, dystonia, tingling, altered mental status, disorientation, memory loss, dizziness, vertigo, acute hearing loss, loss of consciousness and neck pain are potentially caused by intracranial processes. A tentative diagnosis should be traced seriously and with consequence. Hemi facial spasm and palsy as clinical presentation of intracranial meningiomas are reported [3]. Surgical treatment is considered as golden standard. Radiotherapy is indicated in cases the primary tumor was not removed totally or in cases of relapse [4].

\section{Case presentation}

A 27-year-old woman presented with fractured upper central incisors in June 2005 after a bicycle accident (Fig. 1). Clinical examination revealed an extreme left-sided mandibular deviation when opening the jaw, in combination with atrophy of the left masseter muscle. The lower left portion of the face was nearly devoid of tactile sensibility. The symptoms were attributed to a surgical procedure, performed in 1999 for a meningioma of the left cavernous sinus.

The patient has been experiencing recurrent headaches from childhood onwards. The pain radiated to the region 


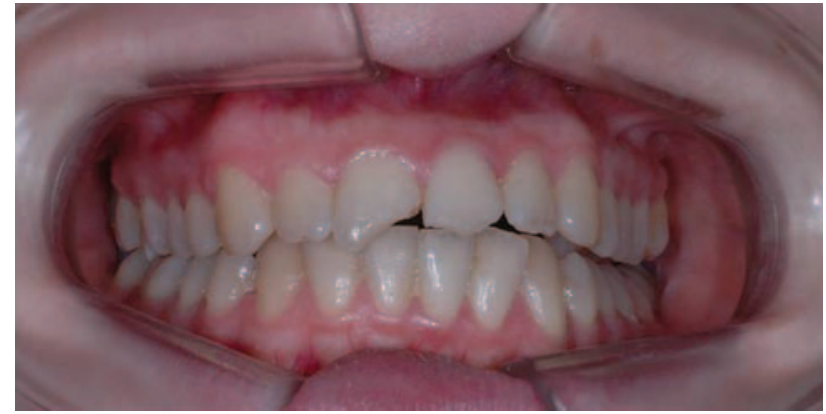

Fig. 1: Intraoral situation after a bicycle accident in 2005

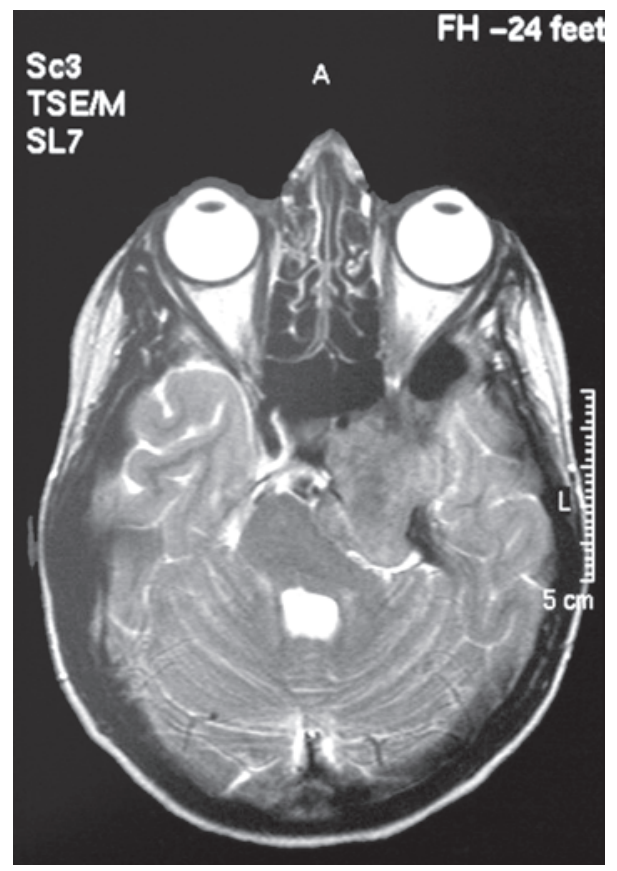

Fig. 2: Nuclear Spin Tomography (1999) showing the large meningeoma in the left cavernous sinus

behind the left eye, with incidental swelling of the periorbital region. In 1997 she noticed dysesthesia and hypalgesia in the left face, mainly in the regions affected by the second and third trigeminal branch. The symptoms worsened and the patient experienced dizziness as well as left-sided weakness of the chewing muscle during this time.

A nuclear spin tomography was performed in June 1999 (Fig. 2). The large meningioma in the left cavernous sinus was removed in July 1999. The trigeminal nerve was preserved and protected from damage as far as possible. No complications have been mentioned in the surgery report. Six days after surgery, the patient experienced an epileptic state without initial aura. The epilepsy occurred because of epidural hemorrhage during trepanation. Immediate surgery was performed and the bleeding was stilled. A fortnight after this second intervention, a rhinoliquorrhea appeared in combination with consecutive meningitis. The cerebrospinal fluid fistula was closed by a surgical procedure. No relapse of the primary tumor was found on magnetic resonance investigations, which were performed at yearly intervals. According to the medical records, in May 2002 the patient developed increasing atrophy of the left masticatory muscles, innervated by the third ramus of the left (motor portion) trigeminal nerve. The disturbance of sensibility in the left face involved the second and third trigeminal nerve. In June 2004 a disturbance was noted in the left temporomandibular joint. Orthodontic treatment with a removable appliance was conducted and the patient was asked to perform myofunctional exercises. In addition physiotherapy was performed, without being effective at this time. Alternative treatment methods also yielded no success.

Functional problems and subjective complains aggravated during the following months. Especially chewing efficiency diminished constantly. In March 2006 the patient presented with cumulative pain in the right joint.

\section{Clinical functional analysis}

Extensive diagnostic procedures, based on our interdisciplinary concept of diagnosis, were started in March 2006. The purpose was to make a conclusive diagnosis of the primary and secondary causes, develop a treatment concept that would decelerate the decompensation of functions of the stomatognathic system (which existed mainly on the left side, but increasing decompensation was noted in the right TMJ as well), and find possibilities to improve the function of the paralyzed left side.

Dysesthesia and hypalgesia were observed in the left half of the face, corresponding to the second and third trigeminal branch. A small area on the Gonion angle, measuring approximately $2 \mathrm{~cm}$ in diameter, was sensitive. The patient's main concerns were to eliminate the pain in the right jaw joint and improve her chewing ability. Her dental history revealed numerous complaints such as pain in the right jaw, headache and spasms in the head and neck (Fig. 3). Muscle palpation was performed only on the right side because of neurological impairment on the left side. The medial pterygoid muscle and the retromaxillary region were painful on palpation. Palpation of the retro-maxillary region is used as an indicator for the inferior head of the lateral pterygoid muscle. The lateral pole

\begin{tabular}{|l|l|l|c|}
\hline \multicolumn{5}{|c|}{ Special Medical Anamnesis (March 2006) } \\
\hline Infections & No & Urogenital Problem & No \\
\hline Cardio-Vascular System & No & Central Nervous System* & Yes \\
\hline Respiratory System & No & Psychological Problem** & Yes \\
\hline Digestive System & No & Rheumatic Disease & No \\
\hline Metabolic System & No & Hormonal Disease & No \\
\hline Allergies & No & Special Problem & No \\
\hline \multicolumn{5}{|c|}{ *St.p. meningeoma, Trigeminus Paresis * Depression } \\
\hline \multicolumn{5}{|c|}{ Okklusal Index (March 2006) } & Yes & 1 \\
\hline 1 & Do you have problems when you chew? & No & 0 \\
\hline 2 & Do you have problems when you are talking? & Yes & 1 \\
\hline 3 & Do you have problems in closing your teeth properly? & Yes & 2 \\
\hline 4 & Are any of your teeth especially sensitive? & Yes & 1 \\
\hline 5 & Do you have a problem when you open your mouth wide? & No & 0 \\
\hline 6 & Do your jaw joints make noise and if so, on what side? & Yes & 2 \\
\hline 7 & Do you have pain in the area of your jaw joints? & Yes & 3 \\
\hline 8 & Do you suffer from headache? & Yes & 3 \\
\hline 9 & Do you suffer from cramps in your head-neck-throat? & Yes & 3 \\
\hline 10 & Do you have in general problems with you posture? & $\mathbf{1 6} / \mathbf{8}=\mathbf{2}$ \\
\hline
\end{tabular}

Fig. 3: Special medical analysis including occlusal index 3/2006 


\begin{tabular}{|l|c|c|}
\hline \multicolumn{3}{|c|}{ Muscle Diagnosis (March 2006) } \\
\hline & Right & Left \\
\hline Shoulders and neck & $\mathrm{X}$ & \\
\hline Atlanto occipital region & & \\
\hline Anterior temporal muscle & & \\
\hline Medial temporal muscle & & \\
\hline Posterior temporal muscle & & \\
\hline Superficial head of masseter muscle & & \\
\hline Deep head of masseter muscle & & \\
\hline Tuber of Maxilla & $\mathrm{xx}$ & \\
\hline Medial pterygoid muscle & $\mathrm{xx}$ & \\
\hline Mylohyoid muscle & & \\
\hline Anterior Head of digastric muscle & $\mathrm{x}$ & $\mathrm{x}$ \\
\hline Supra- and infrahyoid muscles & & \\
\hline Sterno-cleido-mastoid muscle & & \\
\hline Tongue & & \\
\hline \multicolumn{2}{|c|}{ Jaw Joint (March 2006) } & \\
\hline Lateral poles, statically & $\mathrm{x}$ & \\
\hline Lateral poles, in rotation & $\mathrm{x}$ & \\
\hline Retro-condylar joint space & $\mathrm{x}$ & \\
\hline Temporo-mandibular ligament & $\mathrm{x}$ & \\
\hline
\end{tabular}

Fig. 4: Muscle diagnosis and jaw joint examination 3/2006

of the right condyle was painful on static and dynamic (during rotation) examination. Palpation of the retrocondylar space was painful (Fig. 4).

Intraoral inspection showed symmetric dental arches without missing teeth, including the third molars. Apart from marginally crowded front teeth, the arches were well aligned. The molars and the second premolars of the upper and lower jaw were restored with occlusal glass-ionomer cement fillings. The fillings of the right upper first molar, the left upper third molar, the left lower first and third molar, and the right lower

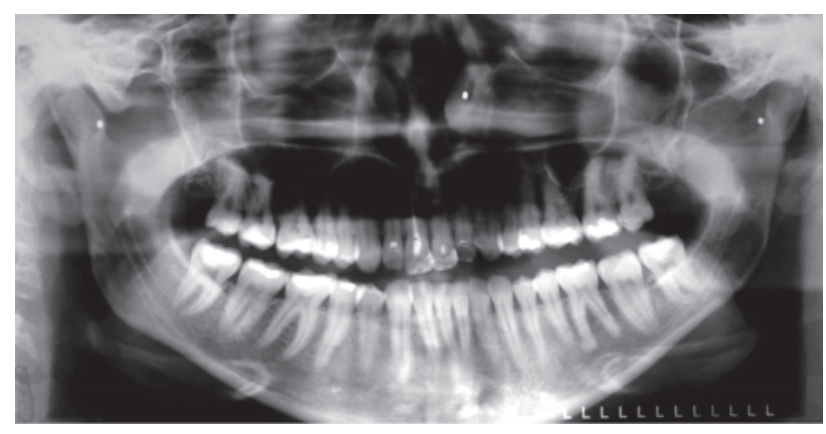

Fig. 5: Panoramic X-ray 3/2006

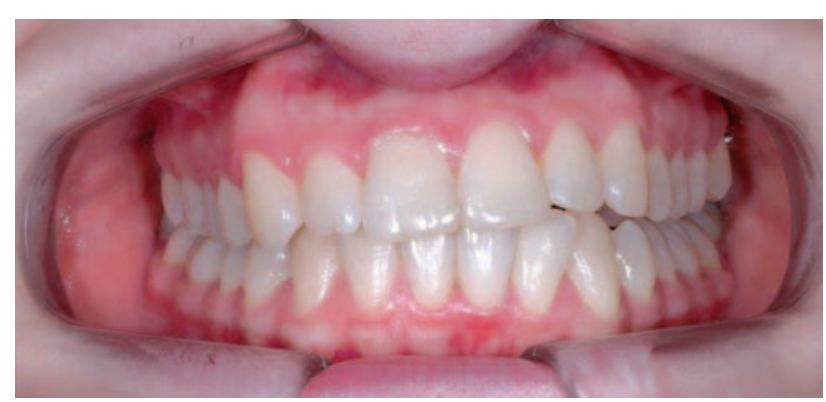

Fig. 6: Intraoral view, frontal aspect, 3/2006 second molar included the cusps. Class I was found on the right side, and non-occlusion on the left side. A tendency to Class I was present on the left side as well. At first sight the nonocclusion on the left side was attributed to an irregularity

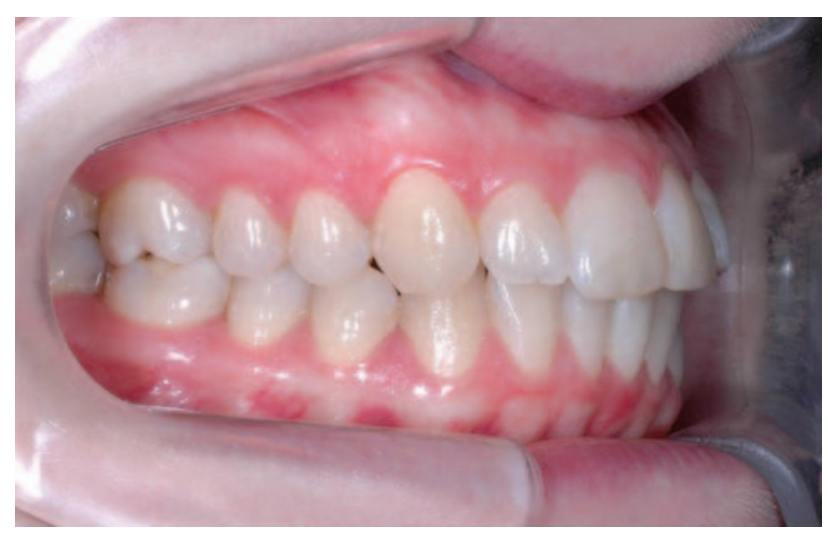

Fig. 7: Intraoral view, right lateral aspect, 3/2006

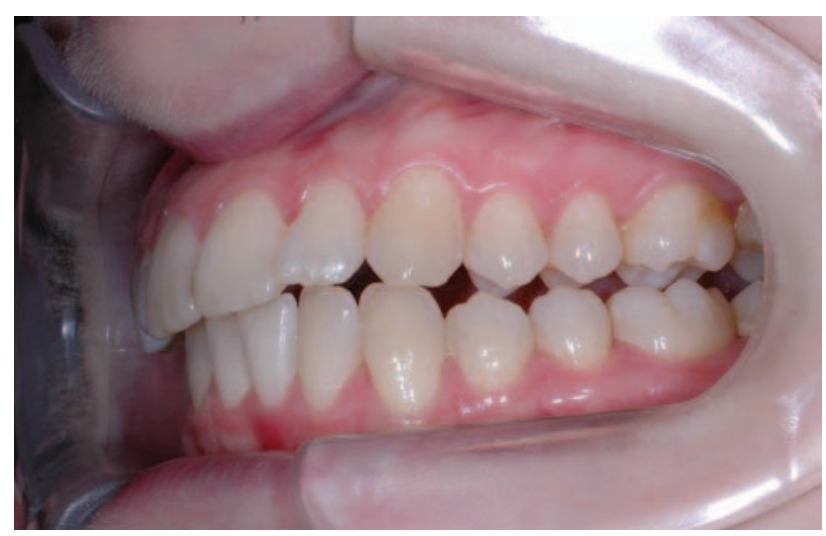

Fig. 8: Intraoral view, left lateral aspect, 3/2006

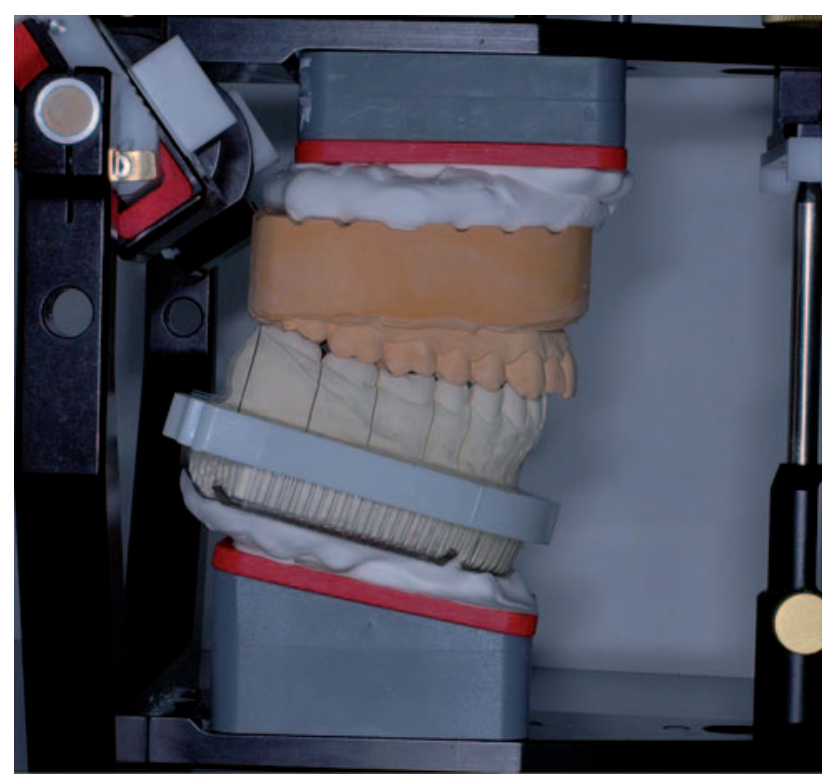

Fig. 9: Casts, mounted in Reference Position (RP), right side 


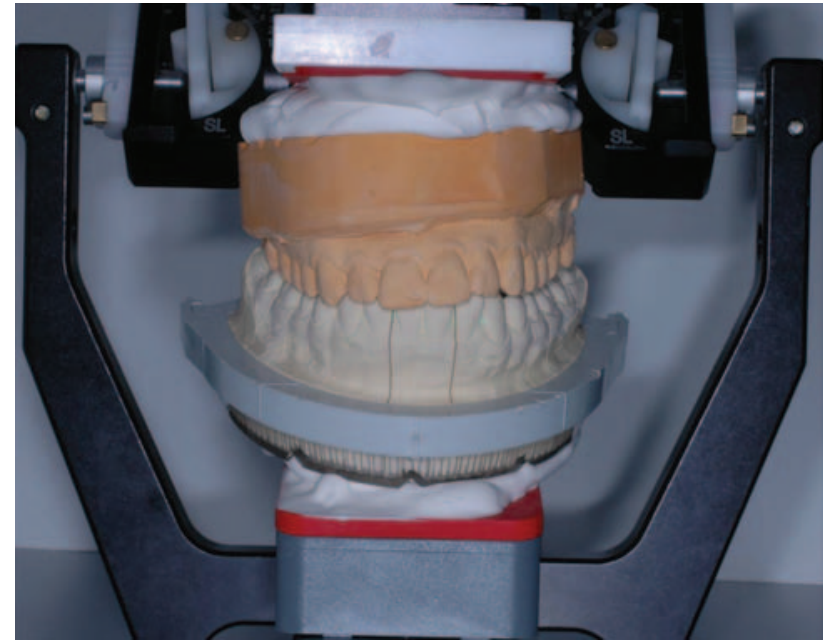

Fig. 10: Casts, mounted in Reference Position (RP), frontal view

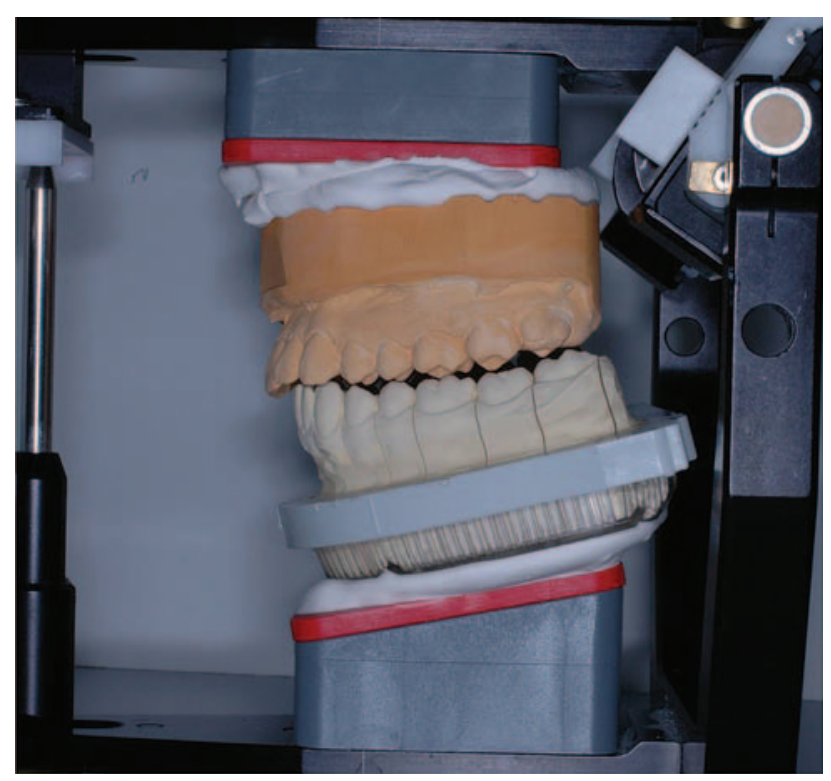

Fig. 11: Casts, mounted in Reference Position (RP), left side
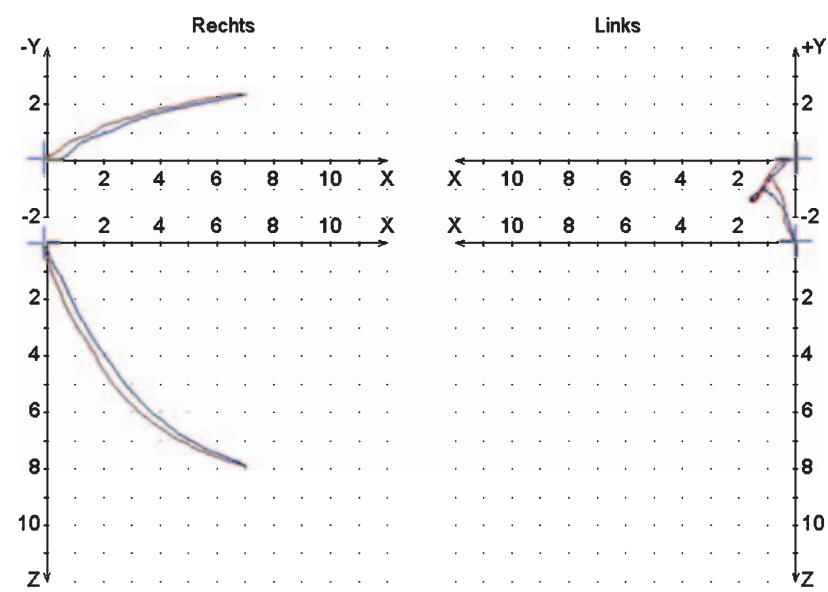

Fig. 12: Initial Condylographic tracing, protrusion-retrusion between the upper and the lower dental arches. Carious lesions, apical processes or other noticeable features were not seen on the panoramic X-ray (Fig. 5). The upper central incisors had been subjected to endodontic treatment because of trauma (bicycle accident in 2005).
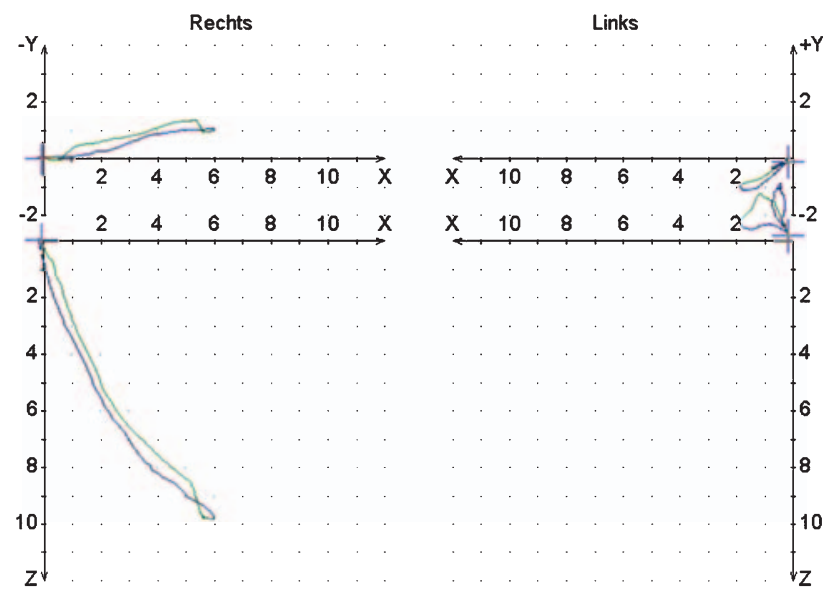

Fig. 13: Initial Condylographic tracing, open-close

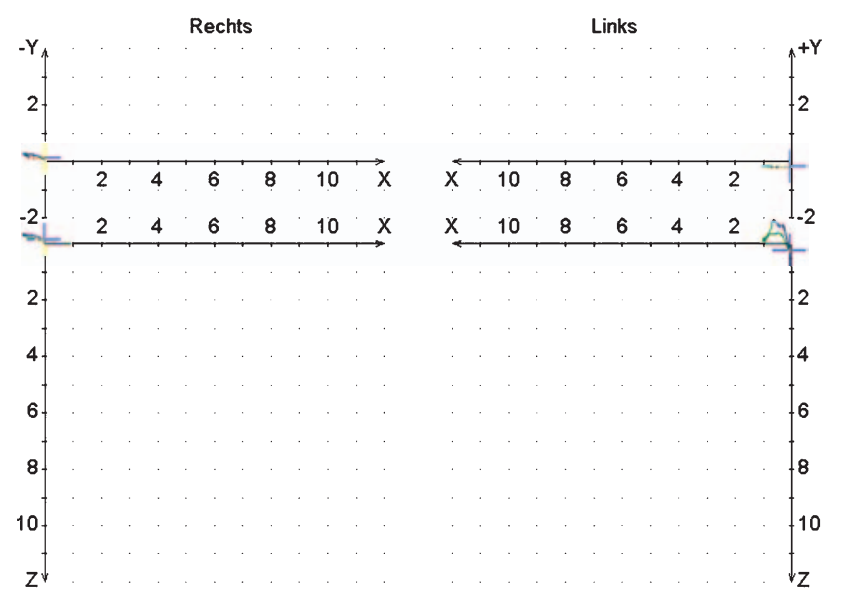

Fig. 14: Initial Condylographic tracing, Mediotrusion left
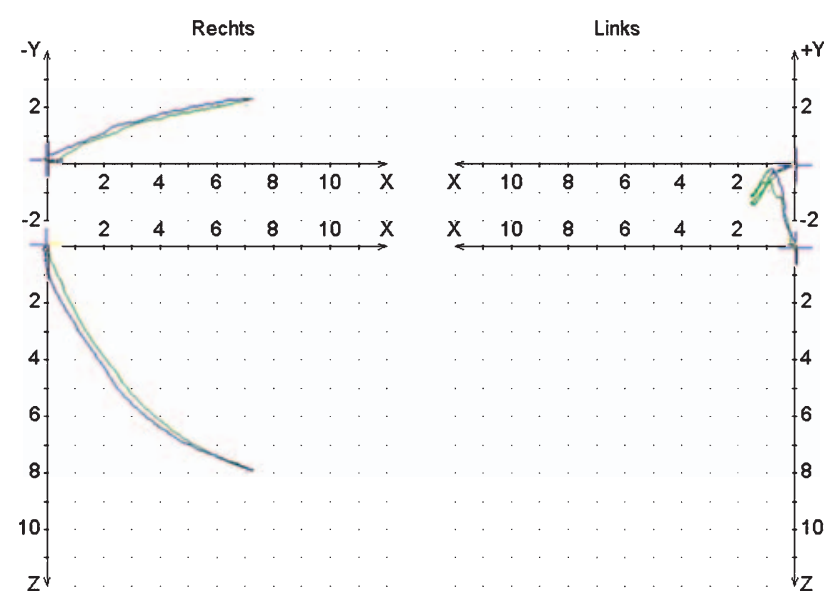

Fig. 15: Initial Condylographic tracing, Mediotrusion right 
Casts of the upper and lower jaw were fabricated and mounted on an articulator (Reference SL from Gamma Dental Company) using an anatomical facebow and centric recording. Non-occlusion was confirmed on the left side. In addition
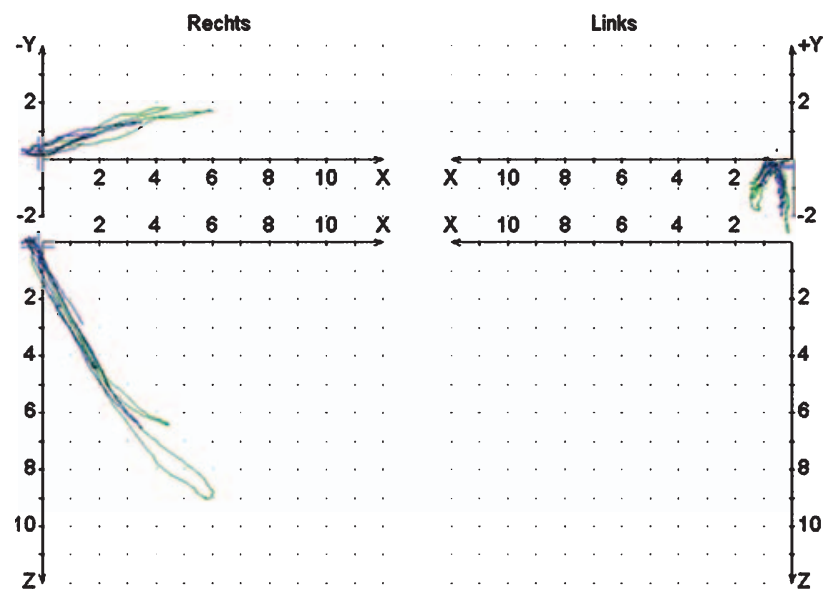

Fig. 16: Initial Condylographic tracing, Speech
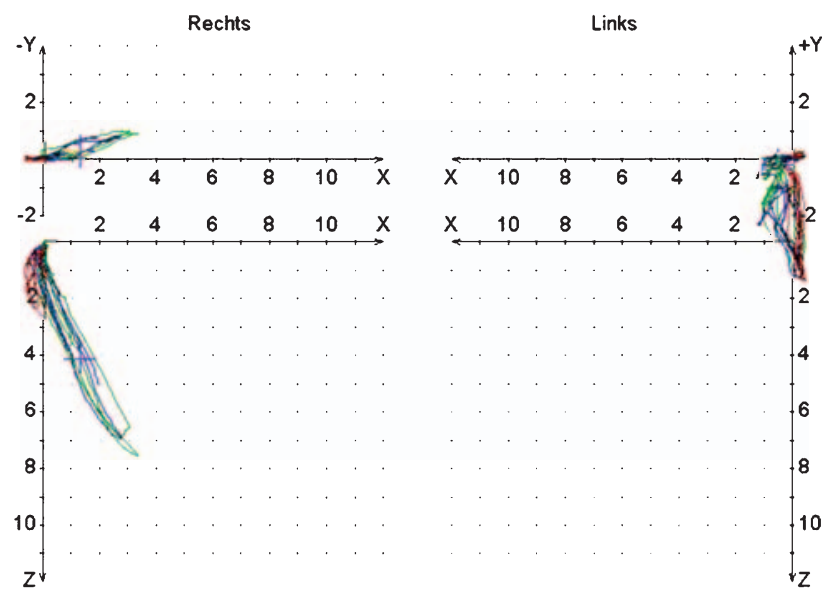

Fig. 17: Initial Condylographic tracing, Mastication
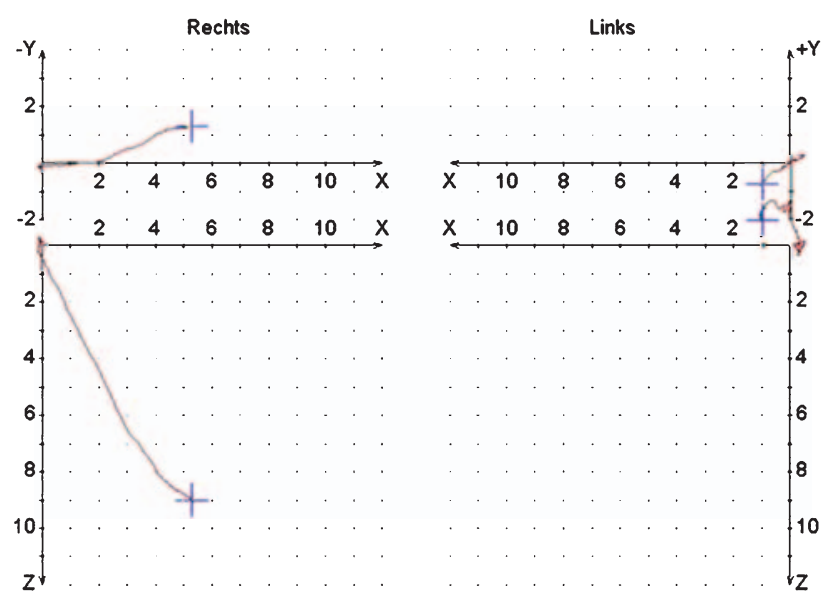

Fig. 18: Initial Condylographic tracing, Deglutation to the intraoral examination, Class I occlusion on the right side was supported only by the lower buccal cusps. However, the lingual cusps were also in non-occlusion (Figs. 6-11).

Condylography was performed using the CADIAX Compact System from Gamma Dental Company. Opening of the mouth, protrusion and mediotrusion were recorded on the left and the right side (Figs. 12-15). In addition, the functional movements of the hinge axis during chewing, swallowing, speech and bruxing were registered (Figs. 16-19). The mobility of the left condyle was greatly reduced. The movement pattern of the left condyle showed a re-, sur- and laterotrusive component. During all mandibular movements,
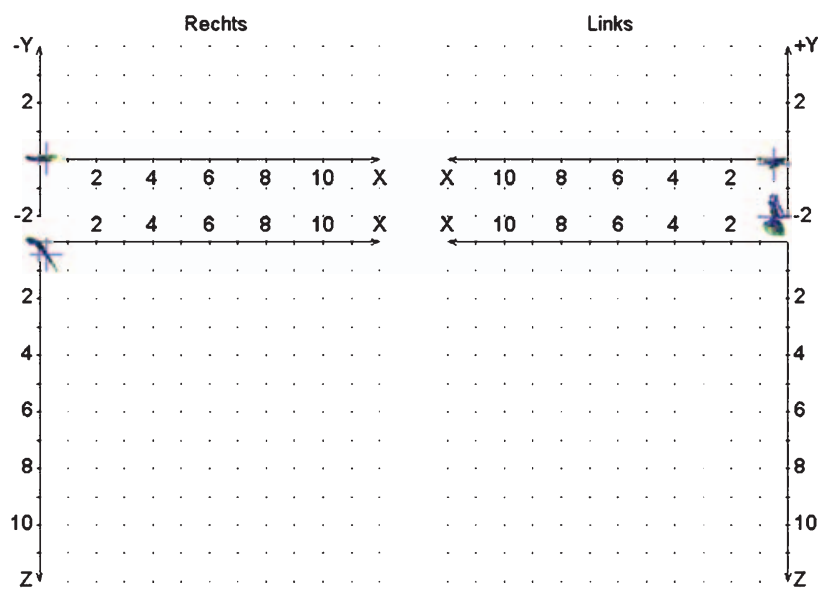

Fig. 19: Condylographic tracing, Bruxing

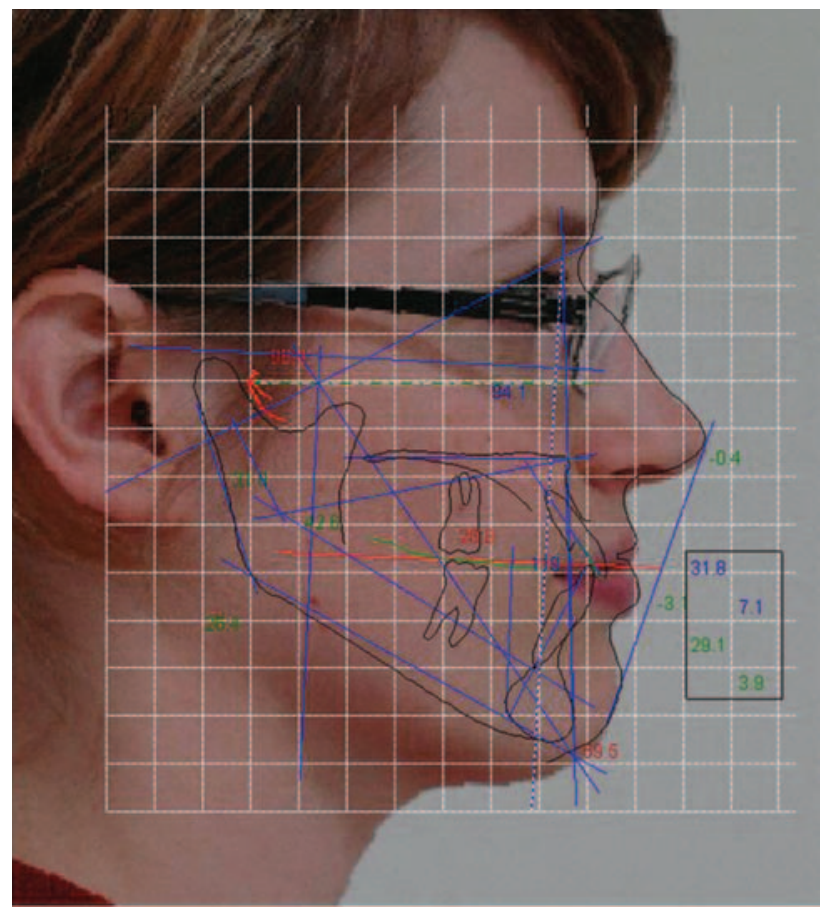

Fig. 20: Lateral Cephalogram: tracing superimposed with profile photography 


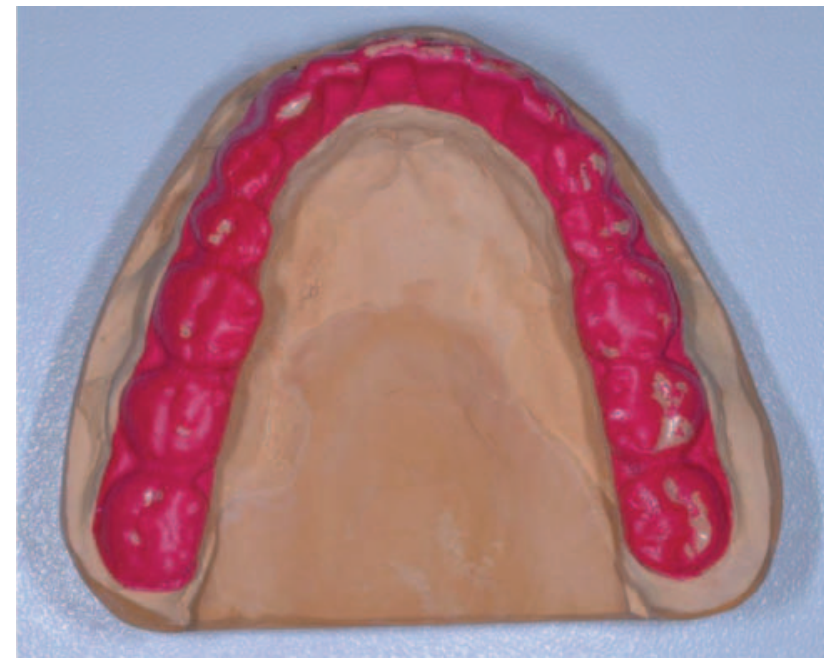

Fig. 21: Bruxing habit, BruxChecker

the motion of the left condyle was less than $2 \mathrm{~mm}$. The magnitude of movements was reduced on the right side as well.

On the cephalogram the skeletal inclination of the skull and the mandible was mesiofacial. A skeletal Class I was found. The maxilla as well as the mandible was positioned prognath. The height of the lower face was normal. Protrusion and inclination of the upper and lower incisors were increased (Fig. 20).

In order to examine occlusion in function, a BruxChecker [8] was fabricated. The patient used this device for one night. The analysis showed bruxing facets only on the right side. Balancing contacts were observed in the second and third molars on the right side (Fig. 21).

\section{Treatment concept}

A concept of treatment was established and discussed with the patient. Psychologically the patient was in a state of depression at this time, but agreed to start the therapy. In principle, the initial phase of the treatment was focused on the neurological situation on the left side. Besides the sensibility deficit of the left trigeminus, the entire motoneuronic portion of the second and third branch of the fifth brain nerve was affected as well. Mandibular movements, such as mastication, swallowing, bruxing and speech were executed only by the chewing muscles on the right side. As a result, cumulative decompensation of the structures on the right side was observed. The outcome of the clinical functional analysis has been described earlier in this report. The patient mentioned a clicking noise which had developed recently and was confirmed by palpation in the right auditory canal. It was concluded that the right jaw joint was overloaded and the disc-condyle relationship had been loosened, exposing the patient to the risk of luxation with, and later without, reduction.

The patient was examined with skin electrodes. Initially the electrodes were placed in the region of the deep and superficial head of left and right masseter, symmetrically on both sides, in accordance with standard EMG protocols (Figs. 22, 23). The absence of any signal on the left side

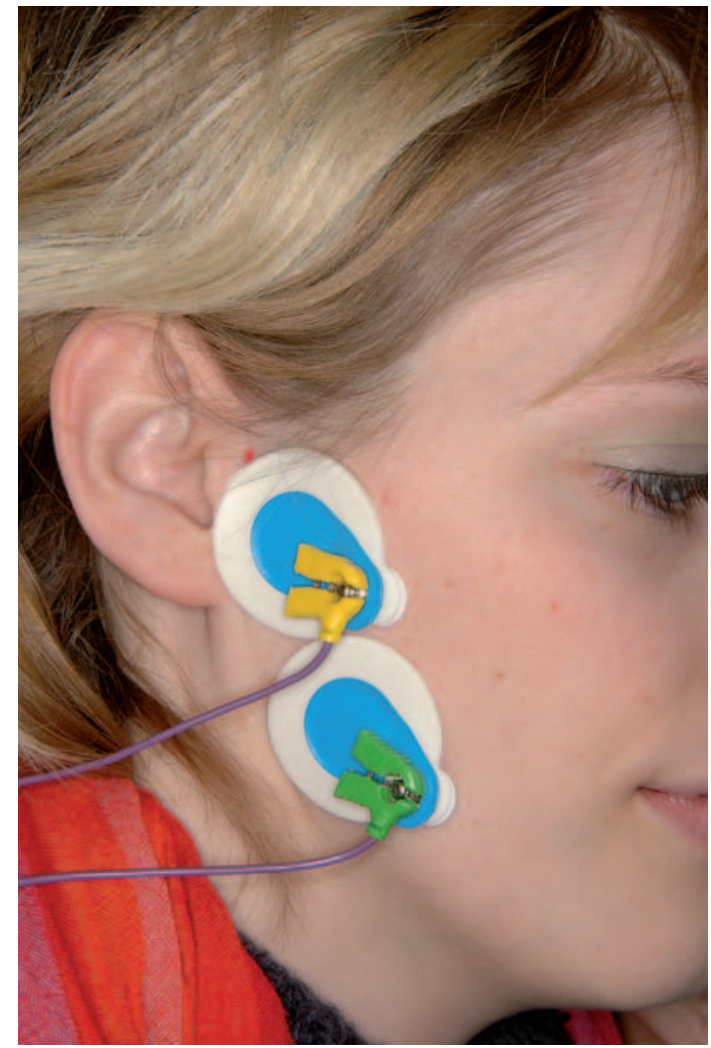

Fig. 22: Placement of the electrodes on the right side

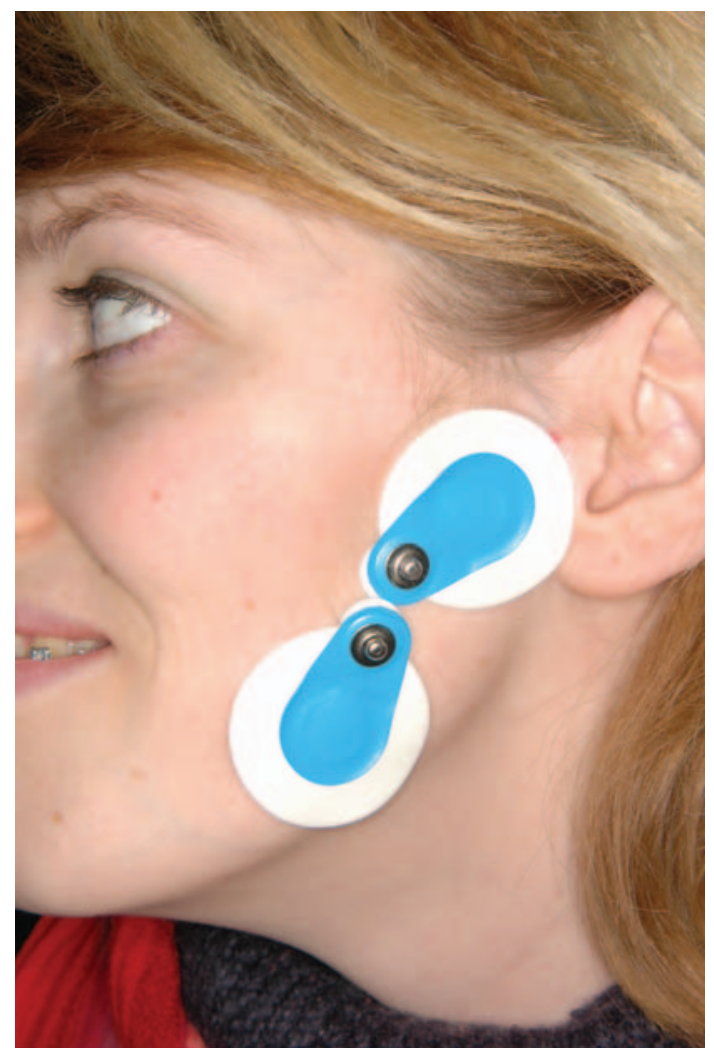

Fig. 23: Initial placement of the electrodes on the left side 


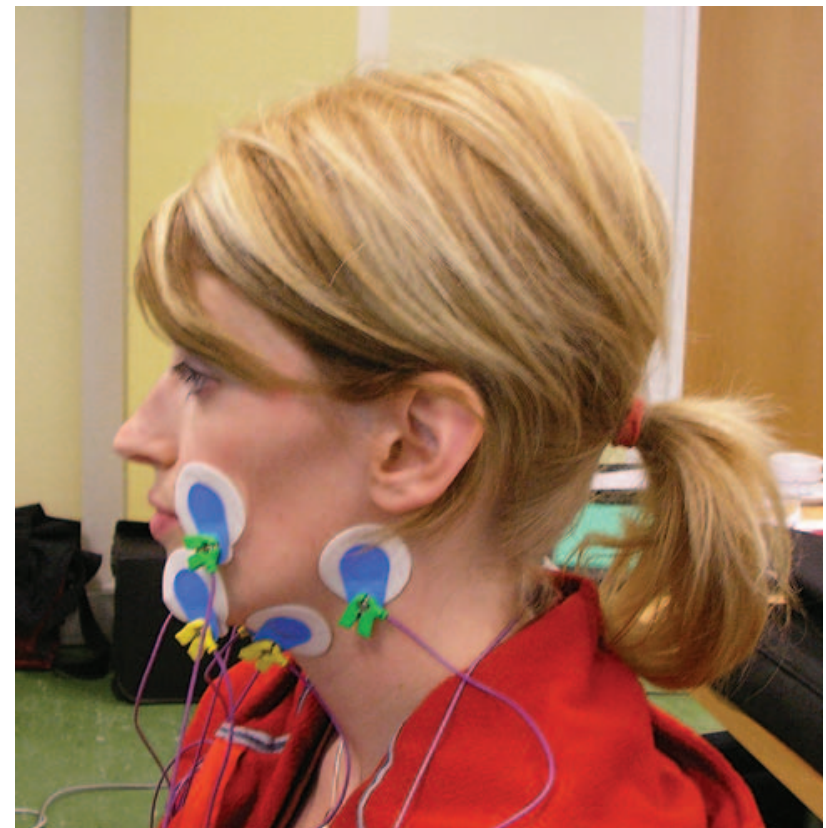

Fig. 24: Demonstration of the alternative placement of the electrodes on the left side

confirmed the tentative neurological diagnosis. The left-sided electrodes were then placed on the facial muscles (Fig. 24). The facial nerve was fully intact and in good order. The platysma, the orbicularis oris muscle and the buccinators on the left side were used to record and demonstrate muscle activity. The position of the electrodes on the right side was not changed. At the first biofeedback therapy session the patient was instructed to activate the facial muscles on the left side and perform coordinated actions together with the right chewing muscle (motor part). She was asked to move the mandible to the right side.

This could be achieved with vehement effort and was accompanied by grimaces. After a training session of thirty minutes the patient was able to perform this exercise satisfactorily under visual control, using a mirror. In addition, the

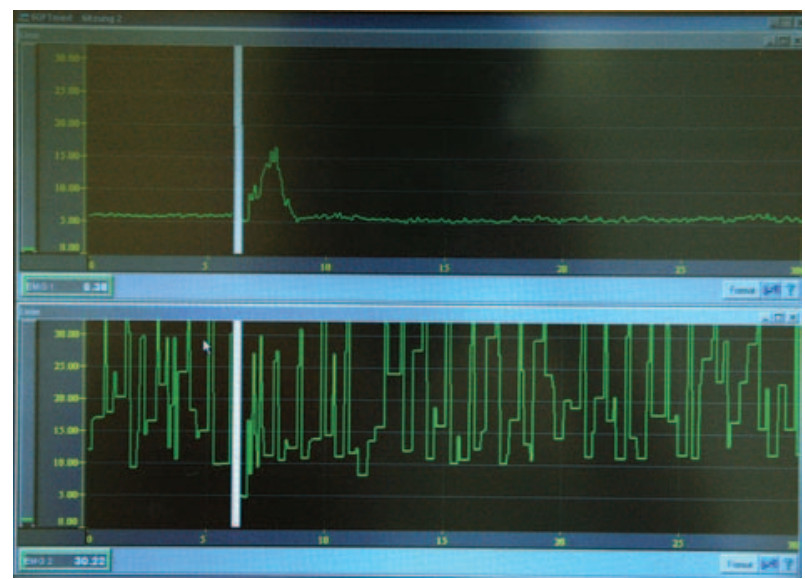

Fig. 25: Initial EMG, the left side is displayed in the upper window patient was challenged to practice chewing. Pieces of apple had to be masticated under conscious and controlled movements on the left and the right side. During the next few days this training program was repeated in front of the computer screen. The patient was then discharged and asked to perform the exercises several times a day without the visual control of the biofeedback system. Two weeks later the left mimic musculature was able to actively support and perform the chewing function with almost no grimaces (Figs. 25, 26).

The patient's mental condition was markedly improved. The exercises were continued for a further two weeks. The entire treatment concept was finalized and discussed with patient. Initially she was asked to wear a nocturnal splint. Replacement of the existing fillings was planned. Orthodontic therapy to establish proper occlusion by closing the left side open bite, followed by final function-oriented moderate restoration, was planned (Figs. 27-30). During the orthodontic treatment which spanned a period of about one year, the condition of the muscles was checked twice. The quantity and quality of coordinated mandibular movements were improved. Daily exercises helped to establish a physiological state in the left joint. The right joint was stabilized again. Signs of overloading and decompensation were successfully inter-

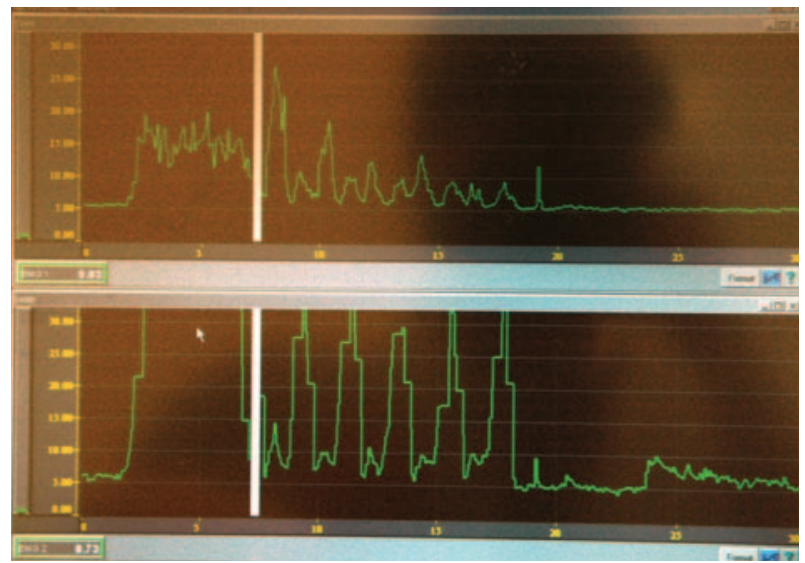

Fig. 26: Follow up EMG, the left side is displayed in the upper window

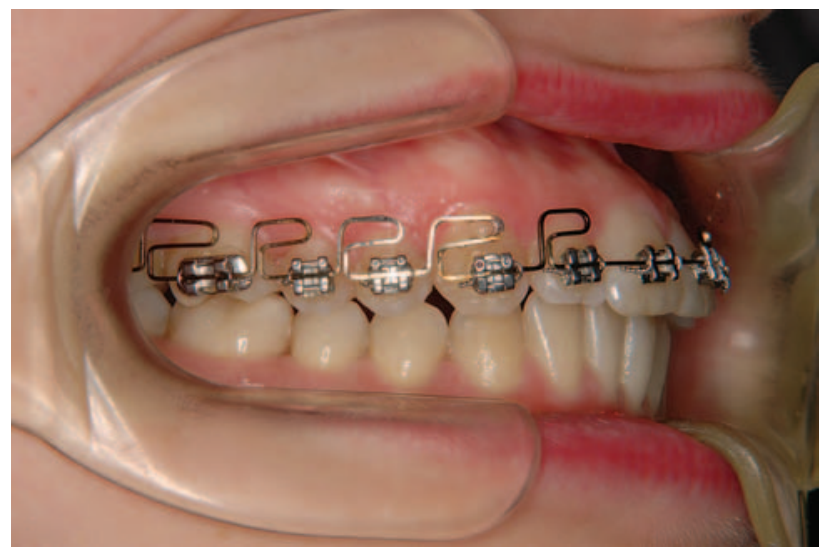

Fig. 27: Orthodontic treatment, right lateral view 


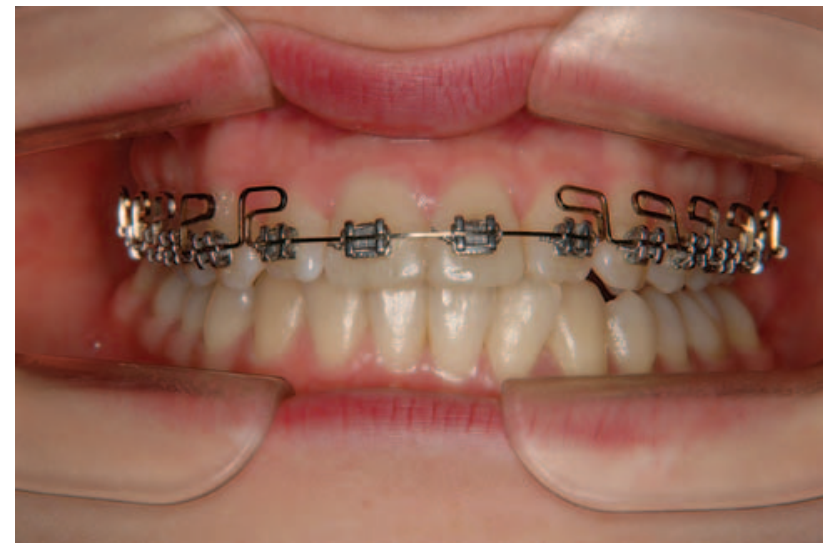

Fig. 28: Orthodontic treatment, frontal view

rupted. Continued exercises were considered essential for stability and long-term success. The patient is highly motivated and continues to perform the exercises.

An ideal occlusion could not be established by the orthodontic treatment. Function-oriented occlusal res-

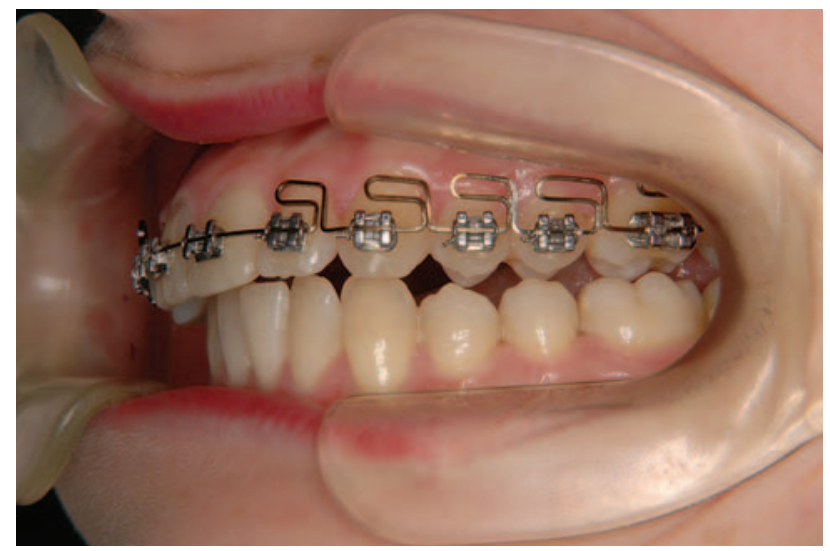

Fig. 29: Orthodontic treatment, left lateral view

toration was performed. The patient will be trained under visual biofeedback control in order to achieve the optimum intercuspidal position. A follow up Condylography showed the improvement of the left joint mobility (Figs. 30-31).
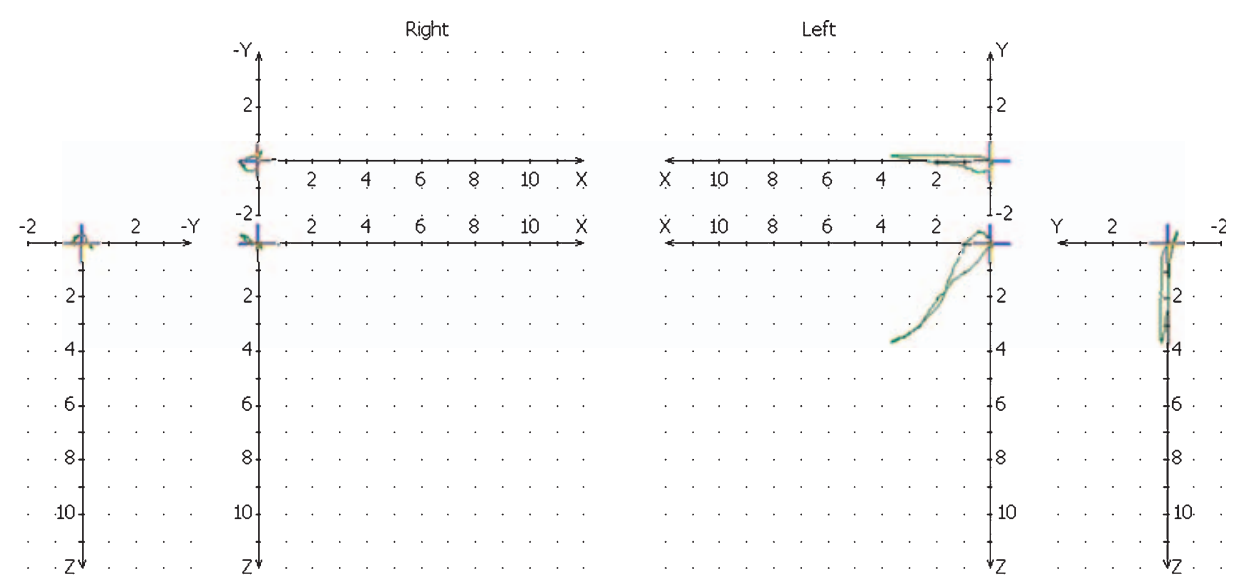

Fig. 30: Follow up Condylography, Mediotrusion left
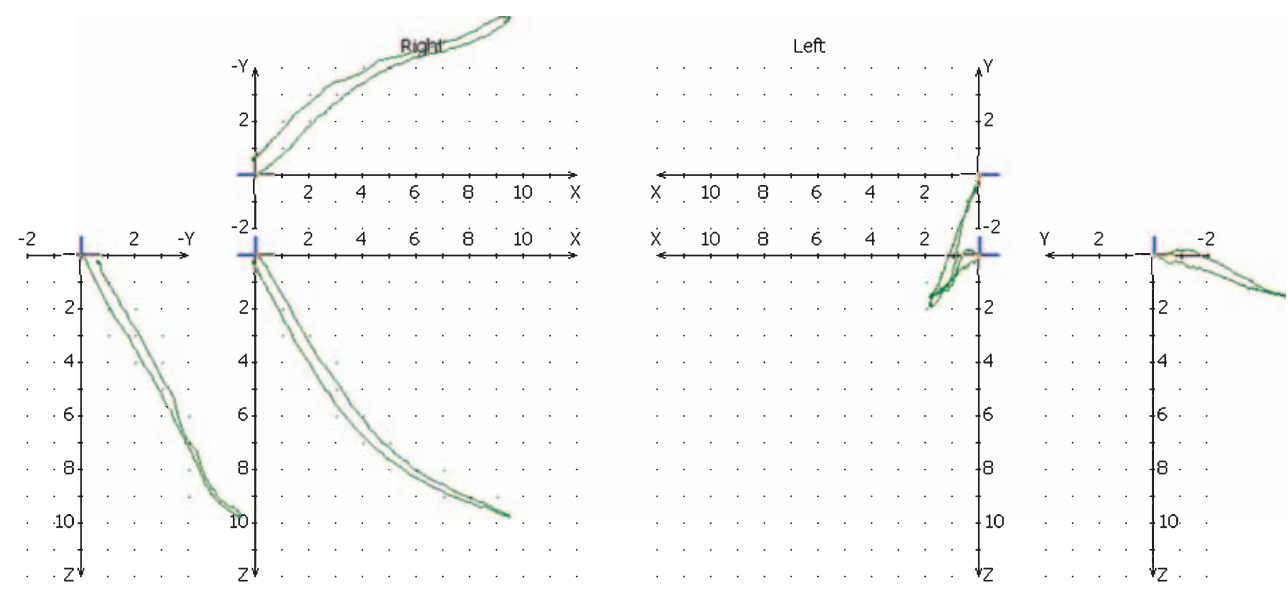

Fig. 31: Follow up Condylography, protrusion - retrusion 


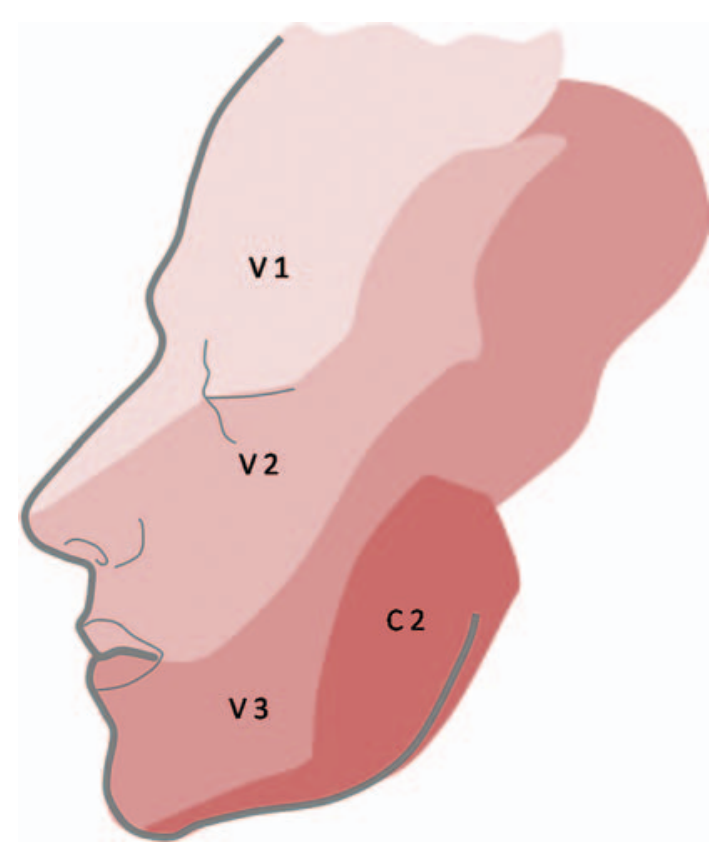

Fig. 32: Sensoric innervations of the face, schematic drawing

\section{Conclusion}

During the treatment period it was found that the sensitivity of the left face had been re-established. At the beginning of the treatment only a small area in the mandibular arc on the left side was innervated. Now the sensitivity extends nearly over the entire lower portion of the left face. It could be explained by the expanded area of N. auricularis magnum (C2) (Fig. 32).

The lower margin of the mandible is supplied to a variable extent by the ventral branch of $\mathrm{C} 2$. At this site the Nervus auricularis magnus extends from the middle of the posterior margin of the sternocleidomastoid muscle, through its lateral surface, upwards in the direction of the ear lobe. A part of it pervades the lobed parotid gland and a posterior branch of it divides and extends to the ear conch as well as its anterior surface. From this site, thin branches supply the angle of the jaw and the cheek. The Nervus cutaneous colli crosses the lateral surface of the sternocleidomastoid muscle and is divided into an upper and a lower branch in the region of the neck. With the descending motor branches of the facial nerve it forms a mixed neural network, known as the Ansa cervicalis superficialis, which supplies the skin of the neck as well as the Platysma.

An interdisciplinary treatment, clearly focused on the recovery of the functions of the stomatognathic system, restores functions and improves local muscular and neuronal properties. The psychic situation of the affected patient is often negatively influenced. The progression of signs and symptoms play an important role and must not be neglected in the care of such patients. To capitulate in such situations cannot be adequate. An active and pre-visional treatment concept has to be implemented. Although a standard treatment is not established for such cases, actions taken have to be arranged on an individual base and have to be implemented with consequence.

\section{References}

[1] Blomquist E, et al. The potential of proton beam radiation therapy in intracranial and ocular tumors. Acta Oncologica 2005;44:862-70.

[2] DeAngelis LM. Brain tumors. N Engl J Med 2001;344:114-23.

[3] Gómez-Perals LF, et al. Espasmo hemifacial como presentación clinica de meningiomas intracraneles. Presentatión de 3 casos y revision de la literature. Neurocirugia 2005;16:21-6.

[4] Jasskelainen J. Seemingly complete removal of histologically benign intracranial meningeoma: late recurrence rate and factors predicting recurrence in 657 patients. A multivariate analysis. Surg Neurol 1986;26:461-9.

[5] Kleihues PDN, et al. The WHO classification of tumors of the nervous System. J Neuropathol Exp Neurol 1993;61(3):215-25.

[6] Mučić-Pucić B, et al. Neurologic symptoms as first signs of brain tumor in children. Acta Clin Croat 2001;40:27-30.

[7] Sieberns J. Analyse des Neurofibromatose 2 Gens in seltenen Meningeomvarianten Dissertation Medizinische Fakultät der Charité Berlin 2006.

[8] www.kdcnet.ac.jp/college/occmed/brux.htm (20 Jan 2008). 